\title{
Carboxy-terminal domain phosphatase 1 silencing results in the inhibition of tumor formation ability in gastric cancer cells
}

\author{
HONGBING FU*, DEJUN YANG* ${ }^{*}$, CHANGMING WANG, JIAPENG XU, \\ WEIMIN WANG, RONGLIN YAN and QINGPING CAI
}

\begin{abstract}
Department of Gastrointestinal Surgery, Shanghai Changzheng Hospital, Second Military Medical University, Shanghai 200003, P.R. China
\end{abstract}

Received October 31, 2014; Accepted August 17, 2015

DOI: $10.3892 / \mathrm{ol} .2015 .3693$

\begin{abstract}
Gastric cancer (GC), one of the most malignant types of cancer, is the second greatest cause of cancer-associated mortality worldwide. Novel therapeutic targets for GC treatment are therefore urgently required. Carboxy-terminal domain phosphatase 1 (CTDP1) has a crucial role in the regulation of gene expression. However, to the best of our knowledge, the role of CTDP1 in GC has not previously been explored. In the present study, reverse transcription-quantitative polymerase chain reaction analysis was used to detect CTDP1 messenger RNA expression in various GC cell lines. CTDP1 was subsequently silenced in GC cells by lentivirus-mediated small interfering RNA (siRNA) infection, and the effects of CTDP1 inhibition on cell proliferation were evaluated by cell number counting, cell cycle analysis with propidium iodide staining and fluorescence-activated cell sorting (FACS) analysis, apoptotic rate with Annexin V staining and FACS analysis, as well as colony formation assay in GC cells. The results revealed that CTDP1 was highly expressed in certain GC cell lines and lentivirus-mediated siRNA infection was able to effectively silence CTDP1 expression in GC cells. CTDP1 inhibition decreased cell proliferation, arrested the cell cycle at G0/G1 phase and increased cell apoptosis in GC cells. Furthermore, the colony formation ability of GC cells was also suppressed by silencing CTDP1. Taken together these results indicated that $\mathrm{CTDP} 1$ has a significant role in the tumor formation ability of GC cells and is a novel and promising therapeutic target for the treatment of GC.
\end{abstract}

Correspondence to: Professor Qingping Cai, Department of Gastrointestinal Surgery, Shanghai Changzheng Hospital, Second Military Medical University, 415 Fengyang Road, Shanghai 200003, P.R. China

E-mail: caiqingpingwcwk@163.com

${ }^{*}$ Contributed equally

Key words: carboxy-terminal domain phosphatase 1, gastric cancer, tumor formation, RNA interference, lentivirus

\section{Introduction}

Gastric cancer (GC) is one of the most prevalent types of malignant cancer, and possesses the second highest cancer-associated mortality rate worldwide (1). Although improvements in hygiene, medical technologies and food preservation techniques have led to a marked decline in the incidence and mortality of GC over the past several decades, the 5-year relative survival rate of patients with GC remains at only $29 \%$, even in the USA (2). Therefore, novel therapeutic methods for surgical management and the novel therapeutic targets for GC treatment remain urgently required.

Although numerous environmental factors, including Helicobacter pylori infection and dietary habits, have significant roles in gastric carcinogenesis, the genetic and epigenetic alterations of multiple genes continue to be considered to have crucial roles in this process (3). Following developments in molecular genetics, carboxy-terminal domain phosphatase 1 (CTDP1) has attracted increasing attention. The CTDP1 gene encodes a phosphatase, FCP1, which is able to dephosphorylate the serine residues of the carboxy-terminal domain (CTD) of the largest subunit of RNA polymerase II, a significant factor involved in gene transcription in eukaryotic cells (4). The CTD phosphorylation level of RNA polymerase II is a crucial element in the regulation of gene expression (5). In addition, FCP1 has phosphatase-independent functions in transcriptional regulation, including as an elongation factor and as a splicing associated factor (6). Although the functions of CTDP1 indicate that it may have an oncogenic role, the majority of research regarding CTDP1 has focused on congenital cataracts facial dysmorphism and neuropathy syndrome, which occurs as a result of CTDP1 deficiency (7). To the best of our knowledge, the detailed role of CTDP1 in tumor development has not previously been studied.

In the present study, the role of CTDP1 in GC cells was investigated. The expression of CTDP1 was detected in various human GC cell lines, and subsequently, lentivirus-mediated small interfering RNA (siRNA) was used to silence the CTDP1 gene in a GC cell line with high CTDP1 expression. The effects of CTDP1 deficiency on the cell proliferation, cell cycle, cell apoptosis and tumor formation ability of GC cells were then evaluated. 


\section{Materials and methods}

Cell culture. Human GC cell lines AGS, BGC-823, SGC-7901, HGC-27 and MGC80-3 were purchased from the American Type Culture Collection (Manassas, VA, USA). BGC-823, SGC-7901, HGC-27 and MGC80-3 cells were cultured in RPMI-1640 medium (catalogue no. 11875093; Gibco Life Technologies, Carlsbad, CA, USA) containing 10\% fetal bovine serum (FBS; catalogue no. 16000036; Gibco Life Technologies) and $100 \mathrm{U} / \mathrm{ml}$ penicillin $-100 \mu \mathrm{g} / \mathrm{ml}$ streptomycin (catalogue no. 15140148; Gibco Life Technologies) at $37^{\circ} \mathrm{C}$ under humidified air containing $5 \% \mathrm{CO}_{2}$. AGS cells were cultured in F-12 medium (catalogue no. 21127022; Gibco Life Technologies) under identical culture conditions.

RNA extraction and reverse transcription-quantitative polymerase chain reaction (RT-qPCR). Total cell RNA was extracted using TRIzol reagent (catalogue no. 15596026; Invitrogen Life Technologies, Carlsbad, CA, USA) according to the manufacturer's instructions. Subsequently, complementary DNA (cDNA) was synthesized using a RevertAid First-Strand cDNA Synthesis kit (catalogue no. K1621; Fermentas; Thermo Fisher Scientific, Pittsburgh, PA, USA). The gene expression levels were examined by RT-qPCR via One Step SYBR ${ }^{\circledast}$ PrimeScript $^{\mathrm{TM}}$ RT-PCR kit II (catalogue no. RR086A; Takara Bio, Inc, Otsu, Japan). PCR cycling conditions were initially performed for $4 \mathrm{~min}$ at $95^{\circ} \mathrm{C}$, followed by 40 cycles at $95^{\circ} \mathrm{C}$ for $10 \mathrm{sec}$ and then by $60^{\circ} \mathrm{C}$ for $30 \mathrm{sec}$. RT-qPCR was performed using a Roche LightCycler 480 system (Roche Diagnostics, Indianapolis, IN, USA). The relative levels of CTDP1 mRNA expression were normalized to the internal control gene, GAPDH. The specific primers were as follows: CTDP1 forward, 5'-ATATGGATCCATGCA AAATCGAGCTCGAGA-3' and reverse, 5'-GCGGCCGCT AATCTTCAATTTACCCTAATA-3'; GAPDH forward, 5'-GAAGGTGAAGGTCGGAGTC-3' and reverse, 5'-GAA GATGGTGATGGGATTTC-3'.

Lentivirus-mediated siRNA gene silencing. The CTDP1 targeting siRNA sequence was 5'-CCCAGTTGCAGA GTAAGAA-3'. The sequence of the scrambled siRNA (SCR-siRNA), which served as a negative RNA interference control, was 5'-TTCTCCGAACGTGTCACGT-3'. The siRNA sequences were inserted into the green fluorescent protein (GFP) expression vector pGCL-GFP (GeneChem Co., Ltd., Shanghai, China). The recombinant virus was packaged using the Lentivector Expression system (GeneChem Co., Ltd), and SGC-7901 cells were infected. Three days subsequently, GFP-positive cells were counted under a fluorescence microscope (IX71 System; Olympus Corp., Tokyo, Japan). The effect of siRNA infection on CTDP1 expression was determined by RT-qPCR analysis on the fourth day. PCR cycling conditions were initially performed for $4 \mathrm{~min}$ at $95^{\circ} \mathrm{C}$, followed by 40 cycles at $95^{\circ} \mathrm{C}$ for $10 \mathrm{sec}$ and then by $60^{\circ} \mathrm{C}$ for $30 \mathrm{sec}$.

Western blotting. Cell lysates were subjected to $8 \%$ SDS-PAGE (Sangon Biotech Co., Ltd., Shanghai, China). The blots were subsequently incubated with rabbit anti-human polyclonal CTDP1 antibody (dilution, 1:2,000; catalogue no. ab137683; Abcam, Cambridge, UK) overnight at $4^{\circ} \mathrm{C}$ and then with secondary antibody (donkey anti-rabbit immunoglobulin $\mathrm{G}$ H\&L horseradish peroxidase-conjugated polyclonal antibody; dilution, 1:10,000; catalogue no. ab16284; Abcam) at room temperature for $2 \mathrm{~h}$ and chemiluminescent substrates (catalogue no. ab5801; Abcam) at room temperature for $30 \mathrm{sec}$. Hybridization with rabbit anti-rat polyclonal anti-GAPDH antibody (dilution, 1:2,000; catalogue no. ab9485; Abcam) was used to confirm equal protein loading.

Cell proliferation assessment. GFP-positive cells with siRNA infection were separated by fluorescence-activated cell sorting (FACS) using a FACSCalibur (BD Biosciences, Franklin Lakes, NJ, USA) according to the manufacturer's instructions. FACS-sorted cells $\left(1.0 \times 10^{3}\right.$ per well) were cultured in 96-well plates, and then observed and counted using a fluorescence microscope (IX71 System; Olympus Corp.) each day for 5 days to assess proliferative ability.

Cell cycle assay. Cells were cultured in 6-well plates for $48 \mathrm{~h}$ and harvested by centrifugation at $200 \mathrm{xg}$ for $5 \mathrm{~min}$ at $4^{\circ} \mathrm{C}$. The cells were washed twice with pre-cooled phosphate-buffered saline (PBS; pH 7.4; Sangon Biotech Co., Ltd.,) and then fixed in $70 \%$ alcohol. Propidium iodide (PI; $50 \mu \mathrm{g} / \mathrm{ml}$; catalogue no. P4864; Sigma-Aldrich, St. Louis, MO, USA) staining was used to determine the percentage of cells at each stage of the cell cycle. The distribution of cells within the cell cycle was analysed by FACS (FACSCalibur).

Cell apoptosis. Those cells exhibiting exponential growth were harvested and stained with allophycocyanin (APC)-labeled Annexin V (catalogue no. 88-8007; eBioscience, San Diego, CA, USA) to detect apoptotic (Annexin V positive) cells. A total of $1.0 \times 10^{6}$ cells were washed twice with pre-cooled PBS (pH 7.4), and incubated for $15 \mathrm{~min}$ in $100 \mu \mathrm{l}$ staining buffer including $5 \mu \mathrm{l}$ APC-labeled Annexin V. FACS analysis for Annexin V staining was subsequently performed by flow cytometry (FACSCalibur).

Colony formation assay. Cells at the exponential growth phase were harvested and re-seeded into 6-well plates at a density of 200 cells/well. The plates were maintained at $37^{\circ} \mathrm{C}$ under humidified air containing $5 \% \mathrm{CO}_{2}$ for two weeks. Following methyl-alcohol fixation with methyl-alcohol (Sinopharm Chemical Regent Co., Ltd., Shanghai, China), the colonies were stained with crystal violet (Beyotime Biotechnology, Haimen, China) for $15 \mathrm{~min}$, followed by using a Sony DSC-H7 Digital camera (Sony Corporation, Tokyo, Japan) and counting the number of colonies per well (visible to naked eye).

Statistical analysis. Data are presented as the mean \pm standard deviation. Statistical significance was determined using Student's t-test with GraphPad Prism 6.01 software (GraphPad Software Inc., La Jolla, CA, USA). P $<0.05$ was considered to indicate a statistically significant difference.

\section{Results}

Lentivirus-delivered siRNA stably inhibits CTDP1 expression in $G C$ cells. Although CTDP1 has a crucial role in gene transcription, studies regarding CTDP1 expression in cancer, and particularly in GC, remain rare. Therefore, the expression 
A

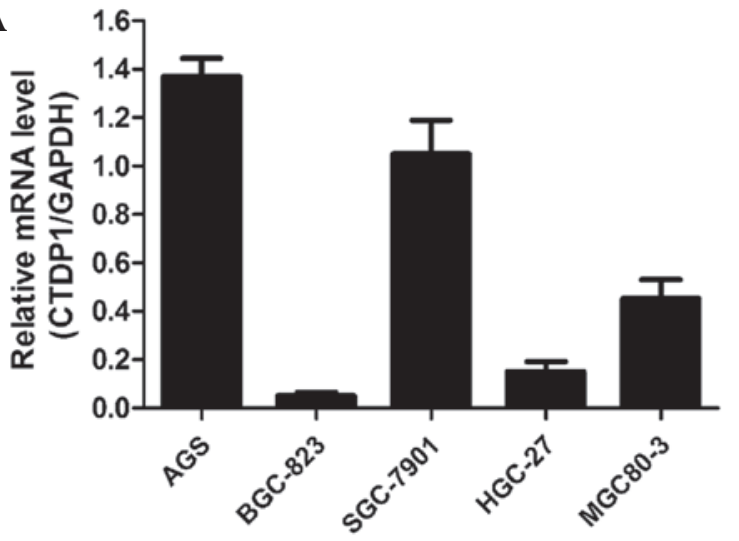

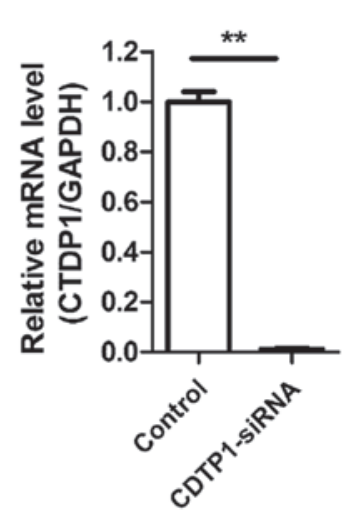

C

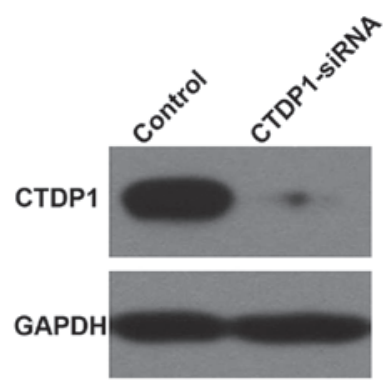

Figure 1. CTDP1 mRNA expression in GC cell lines and siRNA-mediated CTDP1 silencing in SGC-7901 cells. (A) Semi-quantitative detection of CTDP1 mRNA expression (vs. GAPDH) in AGS, BGC-823, SGC-7901, HGC-27 and MGC80-3 GC cell lines by RT-qPCR. The efficiency of siRNA-mediated CTDP1 silencing in SGC-7901 cells was examined by (B) RT-qPCR and (C) western blotting. Control cells were treated with scrambled siRNA infection. Values are expressed as the mean \pm standard deviation. ${ }^{* *} \mathrm{P}<0.01$. mRNA, messenger RNA; GC, gastric cancer; siRNA, small interfering RNA; CTDP1, carboxy-terminal domain phosphatase 1; RT-qPCR, reverse transcription-quantitative polymerase reaction.

A
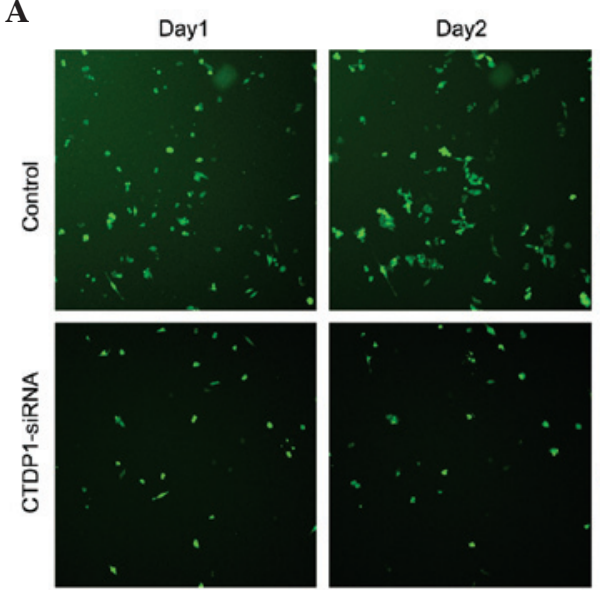

$\mathbf{B}$

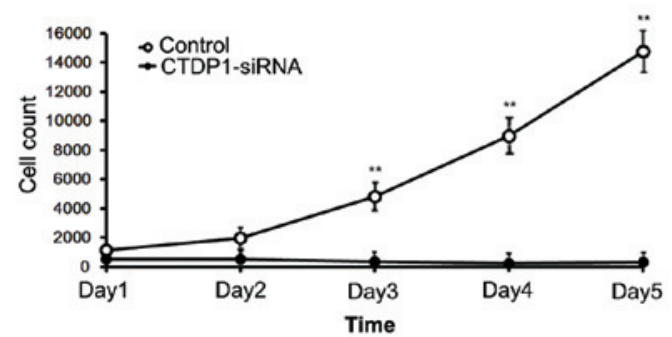

Day3
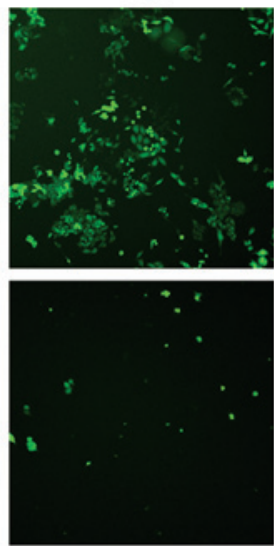
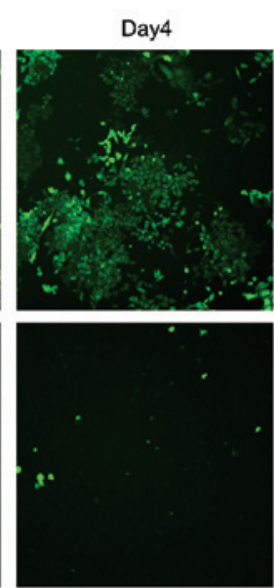

Day5
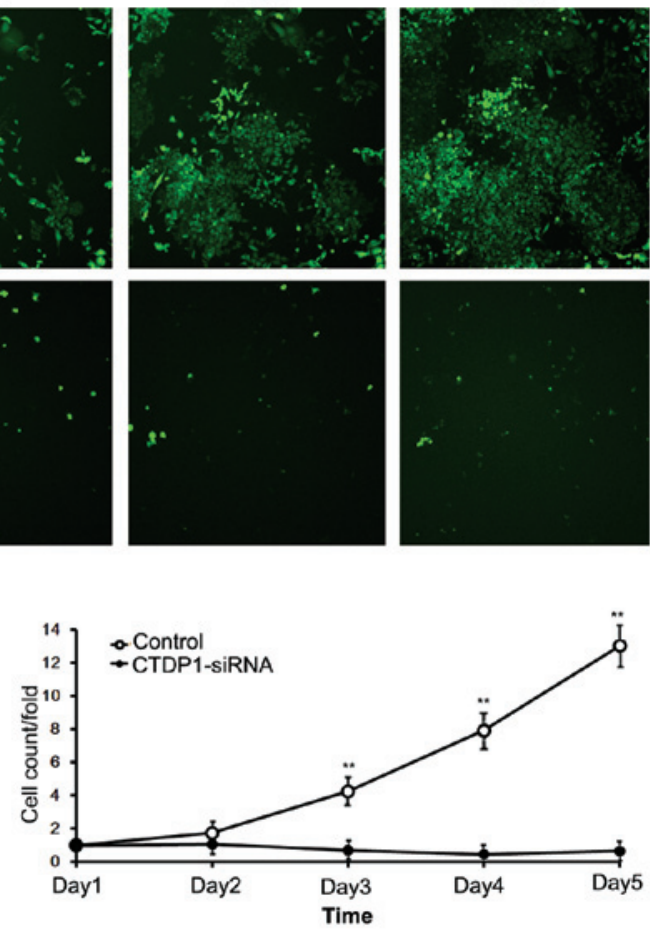

Figure 2. CTDP1 silencing inhibits cell proliferation of SGC-7901 cells. siRNA infected cells expressed green fluorescent protein and were separated and purified by flow cytometry. Cells $\left(1.0 \times 10^{3}\right.$ cells/well) were seeded in 96 -well plates, and the number of cells per well were counted by fluorescence microscopy for 5 days. (A) Representative images (magnification, $\mathrm{x} 100)$ and $(\mathrm{B})$ counting results. Data are presented as mean \pm standard deviation $\left(\mathrm{n}=6\right.$; $\left.{ }^{* *} \mathrm{P}<0.01\right)$. siRNA, small interfering RNA; CTDP1, carboxy-terminal domain phosphatase 1.

of CTDP1 was detected in the AGS, BGC-823, SGC-7901, HGC-27 and MGC80-3 GC cell lines using RT-qPCR. The results revealed that CTDP1 was expressed in all cell lines evaluated, and was particularly highly expressed in the AGS and SGC-7901 cell lines (Fig. 1A). SGC-7901 cells were therefore selected for further analysis of the role of CTDP1 in GC. Lentivirus-delivered siRNA was used to attenuate CTDP1 expression in SGC-7901 cells, and the efficiency of siRNA lentivirus infection was $>90 \%$. Three days following infection, $>90 \%$ of the infected SGC-7901 cells expressed GFP fluorescein. The silencing effectiveness of CTDP1-siRNA on CTDP1 expression was further evaluated by RT-qPCR and western blotting. The results indicated that CTDP1 expression was efficiently inhibited by CTDP1-siRNA infection in SGC-7901 cells (Fig. 1B and C). These data demonstrated that CTDP1 was highly expressed in certain GC cell lines and was able to be effectively and sustainably silenced by lentivirus-delivered CTDP1-specific siRNA.

CTDP1 inhibition decreases cell proliferation ability of SGC-7901 cells. The effects of CTDP1 inhibition on GC cell 
A
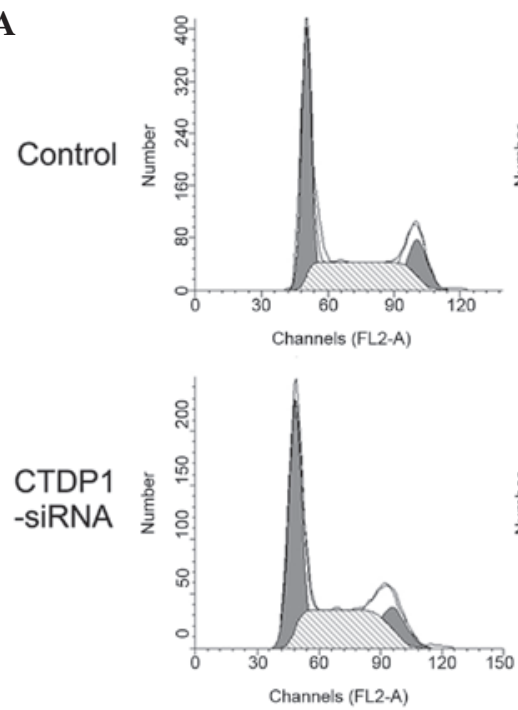
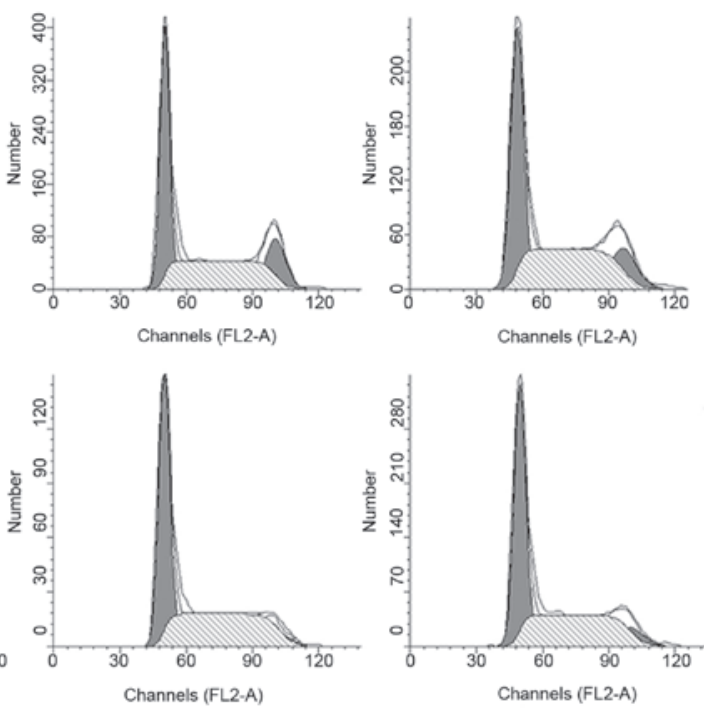

B

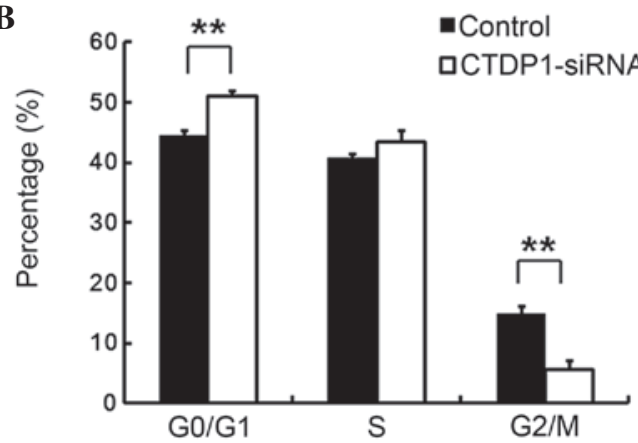

Figure 3. CTDP1 silencing induces cell cycle arrest in SGC-7901 cells. (A) Cell cycle analysis of the cells following siRNA infection was determined by propidium iodide staining and FACS analysis. (B) Percentages of cell cycle phases expressed as the mean \pm standard deviation of three independent experiments, ${ }^{* *} \mathrm{P}<0.01$. siRNA, small interfering RNA; CTDP1, carboxy-terminal domain phosphatase 1.

proliferation. SGC-7901 cells infected with siRNA expressed GFP fluorescein. Therefore, cells that were effectively infected with siRNA were able to be separated by flow cytometry. The separated cells were harvested and monitored for 5 days. The numbers of control cells (infected with SCR-siRNA) increased $\sim 15$-fold in 5 days, while cells infected with CTDP1-siRNA exhibited no significant proliferation, and demonstrated a decrease in cell number (Fig. 2). This result indicated that inhibition of CTDP1 by siRNA infection significantly reduced the cell proliferation ability of SGC-7901 cells $(\mathrm{P}<0.05)$.

CTDP1 inhibition arrests the cell cycle in SGC-7901 cells. In order to investigate the underlying cause of the decrease in cell proliferation in SGC-7901 cells infected with CTDP1-siRNA, the effect of CTDP1 inhibition on the cell cycle was evaluated by PI staining and flow cytometric analysis. The results indicated that cells treated with SCR-siRNA had a lower percentage at G0/G1 phase than that of those treated with CTDP1-siRNA ( $43.7 \pm 1.6$ vs. $51.2 \pm 1.3 \%$; $\mathrm{P}<0.01$ ). In addition, SCR-siRNA-infected cells had $16.3 \pm 1.4 \%$ at $\mathrm{G} 2 / \mathrm{M}$ phase, while the CTDP1-siRNA cells only had $7.5 \pm 2.4 \%$ at $\mathrm{G} 2 / \mathrm{M}$ phase (Fig. 3). These data suggested that CTDP1 inhibition resulted in the arrest of SGC-7901 cells at the quiescent phase.

CTDP1 inhibition promotes cell apoptosis in SGC-7901 cells. The effects of CTDP1 inhibition on cell apoptosis in GC cells was also evaluated using Annexin V-APC staining and flow cytometric analysis. The data revealed that the downregulation of CTDP1 expression resulted in a marked increase in the percentage of apoptotic cells (Annexin V positive) in SGC-7901 cells (from $6.1 \pm 0.4$ to $11.9 \pm 0.7 \%$ ) (Fig. 4). This result demonstrated that CTDP1 inhibition enhanced apoptosis in SGC-7901 cells.

CTDP1 inhibition decreases colony formation ability in $G C$ cells. The aforementioned experiments indicated that inhibition of CTDP1 induced a decrease in cell proliferation ability, cell cycle arrest and an increase in cell apoptosis in SGC-7901 cells. To further investigate the impact of CTDP1 inhibition on GC cells, differences in colony formation ability between CTDP1-siRNA-infected and SCR-siRNA-infected SGC-7901 cells were evaluated. Cells were seeded in 6-well plates at a density of 200 cells/well. Following two weeks of incubation, the SCR-siRNA infected cells exhibited 5.5-fold higher colony numbers compared with those of the CTDP1-siRNA infected cells (Fig. 5). This suggested that CTDP1 inhibition decreased the colony formation ability of SGC-7901 cells.

\section{Discussion}

In the present study, CTDP1 expression was detected in various GC cell lines, CTDP1 expression was stably and efficiently silenced by a specific siRNA-mediated system 
A
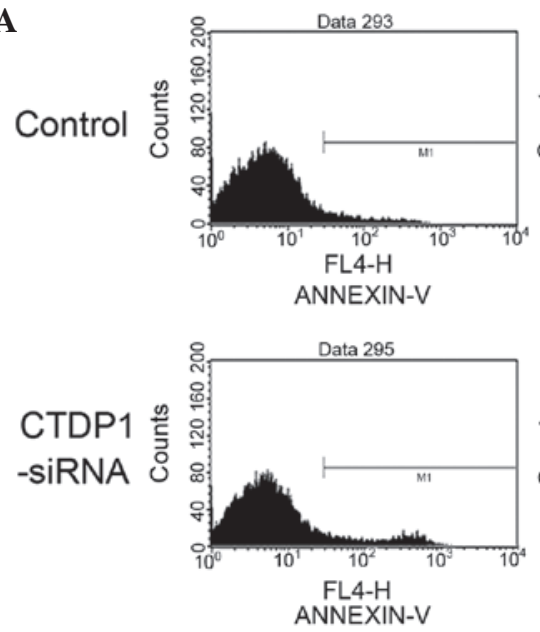
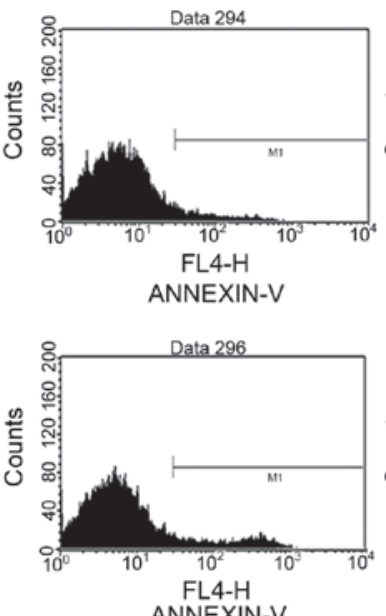

ANNEXIN-V
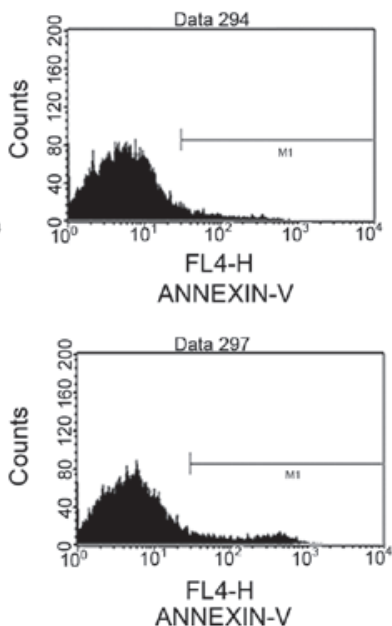

B
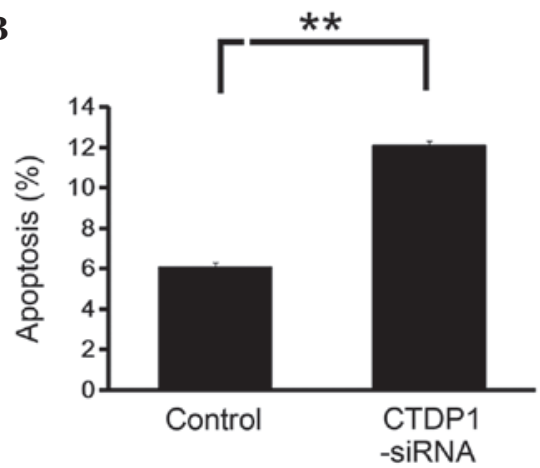

Figure 4. CTDP1 silencing increases cell apoptosis in SGC-7901 cells. (A) Following siRNA infection, the apoptotic level of the cells was evaluated by Annexin V staining and fluorescence-activated cell sorting analysis. (B) Percentages of apoptotic (Annexin V positive) cells are presented as the mean \pm standard deviation of three independent experiments, ${ }^{* *} \mathrm{P}<0.01$. siRNA, small interfering RNA; CTDP1, carboxy-terminal domain phosphatase 1.

A

Control
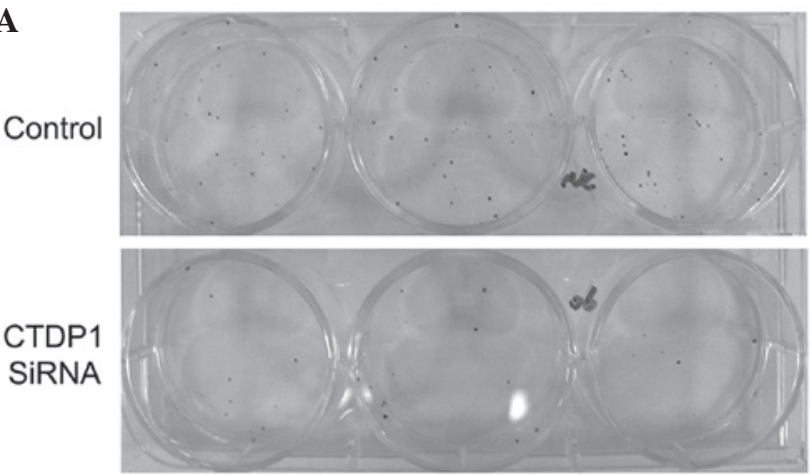

B

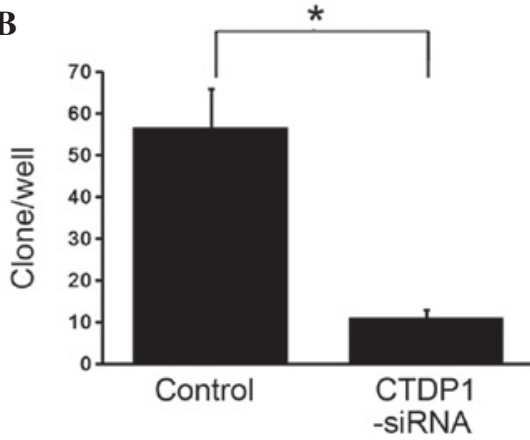

Figure 5. CTDP1 silencing reduces colony formation ability in SGC-7901 cells. (A) Cells with siRNA infection were cultured at a density of 200 cells/well in a 6-well plate for 14 days. Colonies were subsequently stained with crystal violet and counted. (B) Colony numbers are expressed as the mean \pm standard deviation $(n=3),{ }^{*} \mathrm{P}<0.05$. siRNA, small interfering RNA; CTDP1, carboxy-terminal domain phosphatase 1. in SGC-7901 cells, and the effects of CTDP1 inhibition on SGC-7901 cells were evaluated. It was revealed that inhibition of CTDP1 decreased cell proliferation, arrested the cell cycle at G0/G1 phase and increased cell apoptosis in SGC-7901 cells. Furthermore, the colony formation ability of SGC-7901 cells was suppressed by silencing CTDP1. These results suggested that CTDP1 silencing reduced the tumor formation ability of GC cells.

The primary function of FCP1, the protein encoded by CTDP1, is dephosphorylation of the CTD of RNA polymerase II, which has a critical role in the initial synthesis of mRNA and the post-transcriptional modification of mRNA $(8,9)$. The phosphorylation of CTD in the largest subunit of RNA polymerase II mediates the assemblage of regulatory factors during the initial stages of mRNA synthesis $(10,11)$. FCP1 dephosphorylates the CTD of RNA polymerase II in order to recycle it and further initiate a novel round of transcription. Various phosphorylation sites and potential conformational states make CTD a transcriptional controller that is able regulate mRNA production and processing (12).

In addition to regulating RNA polymerase II, FCP1 is also involved in the regulation of RNA polymerase I. Bierhoff et al (13) found that CK2 facilitated a novel round of transcription initiation of RNA polymerase I via the phosphorylation of FCP1. Furthermore, FCP-mediated phosphorylation is not only associated with the assembly of transcription factors, but also temporally controls the cell 
cycle through activation of cyclin-dependent kinases and Greatwall kinase $(14,15)$.

The results of the present study revealed that CTDP1 silencing resulted in proliferation inhibition and cell cycle arrest in GC cells. This indicated that normal expression of CTDP1 may be a decisive factor in cell regulation. Overexpression or silencing of CTDP1 may inhibit its associated regulation mechanisms. Schauer et al (16) found that FCP1 misregulation, whether FCP1 overexpression or silencing, induced p53-dependent and enhanced levels of caspase-mediated apoptosis in Drosophila melanogaster. The present study also found that CTDP1 silencing induced an increase in the apoptotic rate of GC cells.

In conclusion, the results of the present study suggest that CTDP1 silencing is able to suppress cell proliferation, induce cell cycle arrest, increase cell apoptosis and inhibit colony formation in SGC-7901 cells. This finding reveals that CTDP1 has a significant role in GC development, and that CTDP1 may be a promising therapeutic target in the clinical treatment of GC.

\section{Acknowledgements}

The present study was supported by Scientific Research Foundation for Youth Scholars of The Second Military University affiliated Shanghai Changzheng Hospital (no. 2012CZQN12).

\section{References}

1. Torpy JM, Lynm C and Glass RM: JAMA patient page. Stomach cancer. JAMA 303: 1771, 2010.

2. Siegel R, Ma J, Zou Z and Jemal A: Cancer statistics, 2014. CA Cancer J Clin 64: 9-29, 2014

3. Jang BG and Kim WH: Molecular pathology of gastric carcinoma. Pathobiology 78: 302-310, 2011.
4. Chambers RS and Dahmus ME: Purification and characterization of a phosphatase from HeLa cells which dephosphorylates the C-terminal domain of RNA polymerase II. J Biol Chem 269: 26243-26248, 1994.

5. Maniatis T and Reed R: An extensive network of coupling among gene expression machines. Nature 416: 499-506, 2002.

6. Kalaydjieva L: Congenital cataracts-facial dysmorphism-neuropathy. Orphanet J Rare Dis 1: 32, 2006.

7. Varon R, Gooding R, Steglich C, Marns L, Tang H, Angelicheva D, Yong KK, Ambrugger P, Reinhold A, Morar B, et al: Partial deficiency of the C-terminal-domain phosphatase of RNA polymerase II is associated with congenital cataracts facial dysmorphism neuropathy syndrome. Nat Genet 35: 185-189, 2003.

8. Proudfoot NJ, Furger A and Dye MJ: Integrating mRNA processing with transcription. Cell 108: 501-512, 2002.

9. Svetlov V and Nudler E: Basic mechanism of transcription by RNA polymerase II. Biochim Biophys Acta 1829: 20-28, 2013.

10. Dahmus ME: Reversible phosphorylation of the C-terminal domain of RNA polymerase II. J Biol Chem 271: 19009-19012, 1996.

11. Jasnovidova $\mathrm{O}$ and Stefl R: The CTD code of RNA polymerase II: A structural view. Wiley Interdiscip Rev RNA 4: 1-16, 2013.

12. Buratowski S: The CTD code. Nat Struct Biol 10: 679-680, 2003.

13. Bierhoff $\mathrm{H}$, Dundr $\mathrm{M}$, Michels AA and Grummt I: Phosphorylation by casein kinase 2 facilitates rRNA gene transcription by promoting dissociation of TIF-IA from elongating RNA polymerase I. Mol Cell Biol 28: 4988-4998, 2008.

14. Visconti R, Palazzo L, Della Monica R and Grieco D: Fcp1-dependent dephosphorylation is required for M-phase-promoting factor inactivation at mitosis exit. Nat Commun 3: 894, 2012

15. Hégarat N, Vesely C, Vinod PK, Ocasio C, Peter N, Gannon J, Oliver AW, Novák B and Hochegger H: PP2A/B55 and Fcp1 regulate Greatwall and Ensa dephosphorylation during mitotic exit. PLoS Genet 10: e1004004, 2014.

16. Schauer T, Tombácz I, Ciurciu A, Komonyi O and Boros IM: Misregulated RNA Pol II C-terminal domain phosphorylation results in apoptosis. Cell Mol Life Sci 66: 909-918, 2009. 